\title{
Molecular Dynamics Study on Deformation Mechanism of Grain Boundaries in Magnesium Crystal: Based on Coincidence Site Lattice Theory
}

\author{
Ken-ichi Saitoh $\mathbb{D}^{1},{ }^{1}$ Kohei Kuramitsu, ${ }^{2}$ Tomohiro Sato, ${ }^{1}$ \\ Masanori Takuma $\mathbb{D}^{1}{ }^{1}$ and Yoshimasa Takahashi ${ }^{1}$ \\ ${ }^{1}$ Department of Mechanical Engineering, Faculty of Engineering, Kansai University, 3-3-35 Yamate-cho, Suita, Osaka 564-8680, Japan \\ ${ }^{2}$ Mitsubishi Electric Corporation, Japan
}

Correspondence should be addressed to Ken-ichi Saitoh; saitou@kansai-u.ac.jp

Received 10 May 2018; Accepted 24 July 2018; Published 6 August 2018

Academic Editor: Te-Hua Fang

Copyright (C) 2018 Ken-ichi Saitoh et al. This is an open access article distributed under the Creative Commons Attribution License, which permits unrestricted use, distribution, and reproduction in any medium, provided the original work is properly cited.

As for magnesium $(\mathrm{Mg})$ alloys, it has been noted that they are inferior to plastic deformation, but improvement in the mechanical properties by further refinement of grain size has been recently suggested. It means the importance of atomistic view of polycrystalline interface of $\mathrm{Mg}$ crystal. In this study, to discuss the deformation mechanism of polycrystalline $\mathrm{Mg}$, atomistic grain boundary (GB) models by using coincidence site lattice (CSL) theory are constructed and are simulated for their relaxed and deformatted structures. First, GB structures in which the axis of rotation is in [1 100$]$ direction are relaxed at 10 Kelvin, and the GB energies are evaluated. Then, the deformation mechanism of each GB model under uniaxial tensile loading is observed by using the molecular dynamics (MD) method. The present MD simulations are based on embedded atom method (EAM) potential for $\mathrm{Mg}$ crystal. As a result, we were able to observe atomistically a variety of GB structures and to recognize significant difference in deformation mechanism between low-angle GBs and high-angle GBs. A close scrutiny is made on phenomena of dislocation emission processes peculiar to each atomistic local structure in high-angle GBs.

\section{Introduction}

In recent years, improvement of fuel efficiency of the transportation equipment is demanded to reduce burden on the environment. Therefore, there is an issue concerning weight saving of the transportation equipment. For those purposes, one of the materials that attract much attention of researchers now is magnesium $(\mathrm{Mg})$ alloy. $\mathrm{Mg}$ alloy is the lightest metal in practical use and its performance in recycling is noteworthy [1]. Currently this material is used as housing of laptop PCs, cellphones, and so on. However, unfortunately, $\mathrm{Mg}$ alloy still has low workability because anisotropy in plastic deformation is too strong. This is mainly caused by the nature of hexagonal close-packed (HCP) structure that is an elementary crystalline structure of $\mathrm{Mg}$ [2]. Recently, there comes an idea that grain refining process will resolve this problem. This method reduces individual particle sizes in a polycrystal and consequently raises the density of grain boundaries (GBs). Actually, it is reported that both higher strength and ductility are realized by the grain refining [3]. As an effective grain refinement method, severe plastic deformation (SPD) is well known and is paid much attention in the field of plasticity. To name a few, accumulative roll-bonding (ARB) [4], high pressure torsion (HPT) [5], and multidirectional forging (MDF) [6] have been well investigated. Within the above context, we should focus on influence of GB structure on the mechanical properties in the $\mathrm{Mg}$ alloy. Thus, in particular, it is necessary to analyze the influence of GB structure on atomistic deformation mechanism which always takes place in Mg polycrystals. Studies based on molecular dynamics (MD) simulation concerning GBs of $\mathrm{Mg}$ have been extensively reported. For example, relationship among angle of rotation, GB energy, and GB structures is investigated [7], and relationship between the 
deformation mechanism and angle of rotation in tensile or compressive loading is also investigated [8]. However, the view of deformation mechanism in accordance with atomic structure and behavior around GB is insufficient and is still not clear.

Regarding deformation mechanism of Mg crystal, there have been much efforts by many researchers in the world. By using sophisticated experimental apparatus and methods such as electron back-scattering diffraction (EBSD) analyzing technique or transmission electron microscopy observation with atomistic resolution, change of crystal orientation in single crystal and nucleation of defects including twin boundary and dislocation during deformation have been researched extensively [9-12]. However, since experimental and dynamic recognition is quite restricted in this point, some collaborative studies with $\mathrm{MD}$ have been also conducted $[13,14]$. Understanding the mechanism of deformation twinning or dislocation motion in $\mathrm{Mg}$ crystal becomes important recently. It should be noted that, for the purpose of obtaining theoretical insight for such defected structures, there have been a lot of MD studies concerning twinning and dislocations in deformation [15-18]. Within the context of the effect of $\mathrm{GB}$ on plastic deformation of $\mathrm{Mg}$ like in the present study, polycrystalline MD models (though composed of nanosized grains) have been also investigated and those studies are giving many insights as for deformation mechanism around GB region [19-23].

In this study, to analyze the deformation mechanism of polycrystalline $\mathrm{Mg}$, we first produce periodic GB models made up of $\mathrm{Mg}$ crystal in which the axis of rotation is [1 $\overline{1} 00]$ direction, as one of choices. And then, MD simulation of uniaxial tensile loading is performed to observe atomic-scale phenomena around GB. Based on MD results, influence of the GB on nucleation (initiation) and development of slip and twin deformations can be discussed. The relationship between GB energy and GB structures and the relationship between the deformation mechanism and the GB structures in deforming state are discussed too.

\section{Theory and Calculation Method}

2.1. Molecular Dynamics (MD) Simulation and Potential Function. In this study, we use molecular dynamics (MD) method. This method is based on atomic dynamics and is to solve Newton's equations of motion atom-by-atom simultaneously, by numerical integration over time. The basic equation is given by

$$
m_{i} \frac{d^{2} \boldsymbol{r}_{i}}{d t^{2}}=\boldsymbol{F}_{i}=-\frac{\partial \phi}{\partial \boldsymbol{r}_{i}}
$$

where $m_{i}$ is mass of particle $i(i=1 \sim N), \boldsymbol{F}_{i}$ is interatomic force acting on that particle, $\boldsymbol{r}_{i}$ is its position vector, and $\phi$ is interatomic potential energy of total system. When the potential function is formulated among atoms more than two, it is called many-body potential and has high reliability on reproducing materials' properties especially for metallic system. In this study, we use one of many-body potentials, called embedded atom method (EAM) potential [24]. The equation is generally given by

$$
\begin{aligned}
\phi & =\sum_{i} \phi_{i}, \\
\phi_{i} & =\frac{1}{2} \sum_{j(\neq i)} V\left(r_{i j}\right)+F\left(n_{i}\right),
\end{aligned}
$$

where $V\left(r_{i j}\right)$ is a pair potential and $F\left(n_{i}\right)$ is the embedding energy function. $n_{i}$ is individual electron density evaluated at atom $i$ and is determined by contribution from surrounding atoms. Therefore, it is assumed that an expression of electron density function is given by

$$
n_{i}=\sum_{j(\neq i)} \rho\left(r_{i j}\right)
$$

where each $\rho\left(r_{i j}\right)$ is simply pairwise density function as to just two atoms, $i$ and $j$. In this study, we apply an EAM potential function and a parameter set that were proposed for $\mathrm{Mg}$ crystal by Liu et al. [25]. They successfully developed it by fitting to experimental data in bulk, cluster, and liquid state of $\mathrm{Mg}$ as well as some structures including crystalline defects and their energies.

\subsection{Coincidence Lattice Theory Model of Mg Crystal for MD} Simulation. All the GB models of $\mathrm{Mg}$ crystal studied in the present study are constructed by using coincidence site lattice (CSL) theory [26], which has been well known and extensively used in GB studies [27]. It was shown that the CSL theory also can be applied to hexagonal crystal [28]. By using CSL, MD model is adequately applied under periodic boundary condition as shown in Figure 1. In practice, the GB model for MD is constructed as in the following procedure or algorithm. First, $\mathrm{Mg}$ atoms are initially placed in the region of $z>0$, so that $x, y$, and $z$ axes are equal to [11 20$]$, [1 $\overline{1} 00]$, and [0001] directions, respectively (those directions are described in Miller-Bravais indices for hexagonal crystal). Then, that crystal grain is rotated by a certain angle around the $y$ axis, called rotation axis or tilt axis. The angle of rotation is rigorously determined by CSL framework. Subsequently, we place new atoms in another grain, at positions with mirror symmetry with regard to their original counterpart, in $z<0$ region, and consequently we create GB plane along $z=0$. Though, in real materials, there must be asymmetric GBs plane and therefore asymmetric atomic arrangement over GB plane, it is reasonable that we confine our present study to symmetrical GB types, for simplicity.

We choose several rotation angles $\Theta$ between two symmetrically arranged crystals $(\Theta$ angles are measured from each other and usually called misorientation angles). Values of $\Theta$ are calculated according to their own CSL. The CSL is a two-dimensional unit lattice (in the present case it is on $x z$ plane), which is determined by lattice constants $c$ and $a$, together with their axes ratio $c / a=\sqrt{8 / 3}$ which is obtained for ideal hexagonal crystal. The CSL is specified by $\sum$-value, which is a fraction of the number of CSL lattice points to the number of all lattice points in the crystal. As explained above, atomic positions in one grain are rotated by a half of misorientation angle, that is, $\Theta / 2$, on $x z$ plane, and their periodic lattice points per CSL are also placed. Thus, the 
TABLE 1: Geometrical conditions of GB models (As GB model, the most energetically stable one is chosen.)

(a)

\begin{tabular}{lc}
\hline Tilt (rotation) axis & {$[1 \overline{1} 00]$} \\
Lattice constants $[\mathrm{nm}] a, c$ & $0.3202,0.5228$ \\
\hline
\end{tabular}

(b)

\begin{tabular}{lcccc}
\hline Model & Misorientation angle $\Theta$ [deg.] & $\sum$-value & Cell size in $x, y, z[\mathrm{~nm}]$ & The number of atoms \\
\hline A & 11.7 & 97 & $10.30,3.33,25.19$ & 36816 \\
B & 23.1 & 25 & $10.46,3.33,22.35$ & 33240 \\
C & 30.5 & 58 & $9.95,3.33,19.42$ & 27360 \\
D & 44.4 & 14 & $11.07,3.33,20.21$ & 31680 \\
E & 63.0 & 11 & $12.01,3.33,20.79$ & 35424 \\
F & 78.5 & 10 & $10.75,3.33,20.05$ & 30888 \\
G & 88.8 & 49 & $11.21,3.33,21.88$ & 35040 \\
H & 91.2 & 49 & $10.98,3.33,22.37$ & 34848 \\
I & 101.5 & 10 & $10.12,3.33,21.41$ & 30840 \\
J & 117.0 & 11 & $11.04,3.33,23.75$ & 37584 \\
K & 122.9 & 35 & $10.71,3.33,21.84$ & 33168 \\
L & 135.6 & 14 & $11.86,3.33,22.03$ & 37128 \\
M & 146.0 & 35 & $10.94,3.33,21.28$ & 33120 \\
\hline
\end{tabular}

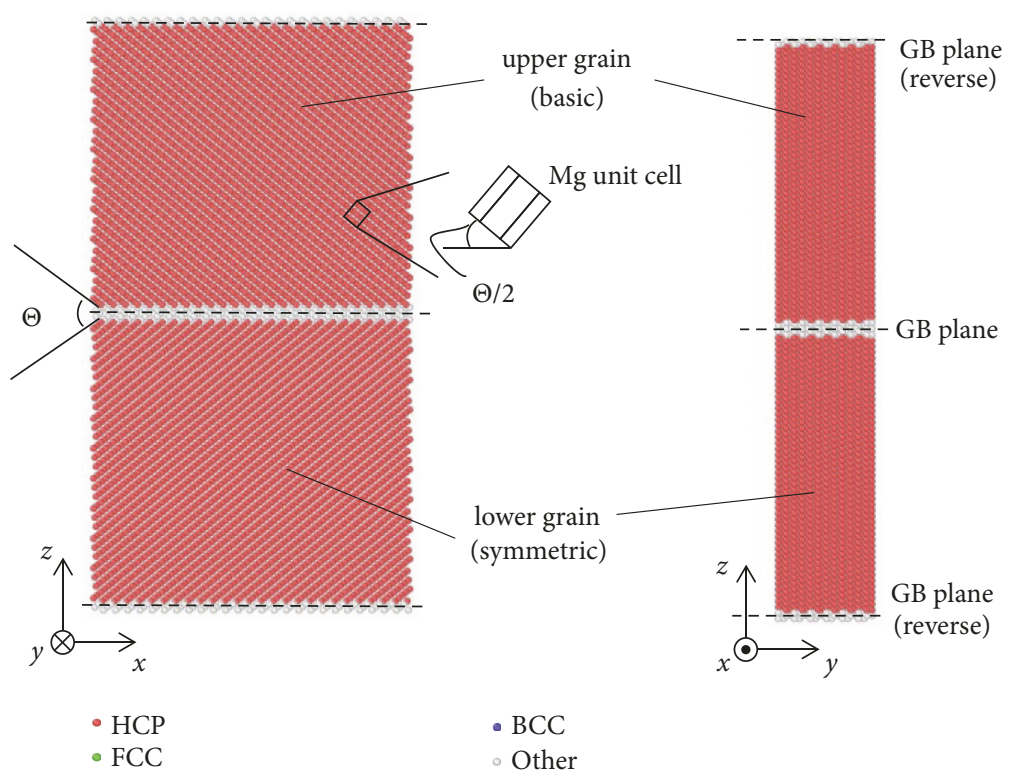

Figure 1: GB model analyzed by CNA.

$x$ - and $z$-sizes of the model should be strictly equivalent to multiple units of CSL size. The misorientation angle $\Theta$ is given by

$$
\Theta=2 \tan ^{-1}\left(\frac{\sqrt{8} n}{\sqrt{3} m}\right)
$$

where both $n$ and $m$ are arbitrary integers. The resulting GB models used here are named by alphabetical label in order of the magnitude of $\Theta$ : Models “A, B, C, D, E, F, G, H, I, J, K, $\mathrm{L}$, and M". Geometrical conditions of GB models (type, size, total number of atoms, etc.) used here are shown in Table 1.
These geometrically obtained initial atomic configurations of periodic GB models are provided adequate structural relaxation by $\mathrm{MD}$ simulation, so that energy and stress components of the total system are just thermally equilibrated. After that, a GB excess energy $\gamma_{\mathrm{GB}}$ can be evaluated using an usual formula given by [27]

$$
\gamma_{\mathrm{GB}}=\frac{1}{2 \times A_{\mathrm{GB}}}\left(E_{\mathrm{GB}}-\frac{N_{\mathrm{GB}}}{N_{\mathrm{pc}}} E_{\mathrm{PC}}\right),
$$

where $A_{\mathrm{GB}}$ is $\mathrm{GB}$ area, $E_{\mathrm{GB}}$ and $E_{\mathrm{PC}}$ are total potential energies of the fully relaxed GB model and a perfect crystal 


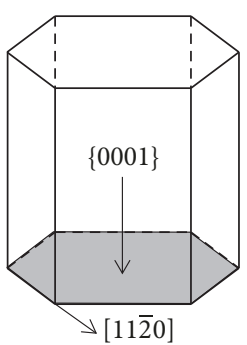

(A) Basal slip

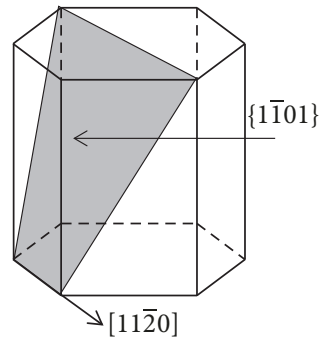

(C) First pyramidal slip

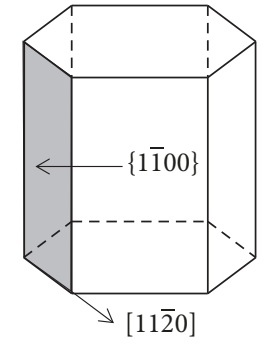

(B) Prismatic slip

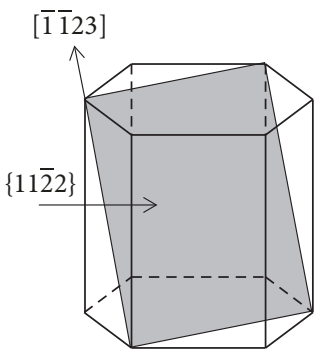

(D) Secondary pyramidal slip

(a) Slip deformation

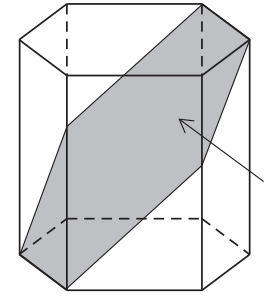

(A) $\{\overline{1} 102\}$ twin

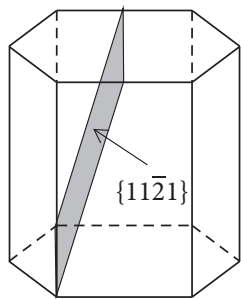

(D) $\{11 \overline{2} 1\}$ twin

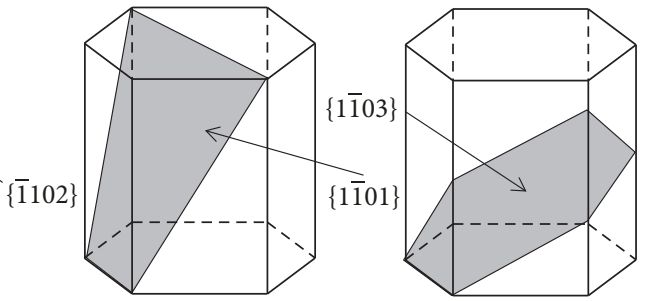

(B) $\{1 \overline{1} 01\}$ twin

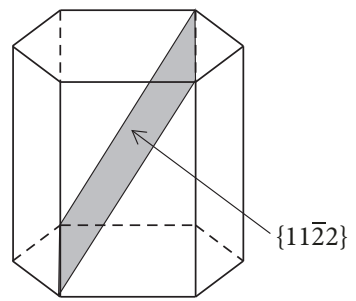

(E) $\{11 \overline{2} 2\}$ twin
(C) $\{1 \overline{1} 03\}$ twin

FIGURE 2: Schematic of deformation mechanism possible in magnesium (Mg) crystal.

(PC) model, respectively, and $N_{\mathrm{GB}}$ and $N_{\mathrm{PC}}$ are the number of atoms in the GB model and the PC model, respectively. In constructing GB models by the above procedure, there are cases when atoms around GB plane are much nearer than an allowable interatomic distance for moderate energy; that is, excessive atoms occur. In such case, excessive atoms are to be removed from the GB model before structural relaxation calculation. Since there are several patterns to remove excessive atoms, several models are created and compared for each $\Theta$. Thus, one structure having the lowest energy is selected for each $\Theta$ and it is regarded as the most stable and the most realistic GB structure.

After structural relaxation, GB models are subjected to tensile loading in the $z$ direction as shown in Figure 1, and deformation behavior of atomic system is observed.

Details of an adaptive tensile loading method were described in [29]. Let me describe the method in brief. Strain rate in tensile direction is prescribed, and then elongation corresponding to strain value at each step is applied in the size of tensile direction. If the cell size were kept constant in other directions, the calculation cell would produce tension also in those directions too. To avoid it and to realize uniaxial tension, size of the calculation cell not in tensile direction is continuously adjusted to avoid normal stress component in that direction. It needs a criterion of tolerance for stress fluctuation, so we set it in the range $\pm 1.0 \times 10^{5} \mathrm{~Pa}$. Thus, the periodic cell is always kept uniaxially being deformed in just one tensile direction.

Structural analysis of atoms is mainly conducted by common neighbor analysis (CNA) [30]. This method is summarized as follows. At first, as to one atom, the bonding state to the first neighboring atoms together with the link state with the 2 nd nearest atoms is observed. Then, comparing
TABLE 2: Calculation conditions for MD simulation.

\begin{tabular}{lc}
\hline Temperature $[\mathrm{K}]$ & 10.0 \\
Relaxation time $[\mathrm{ps}]$ & 50.00 \\
Time step $[\mathrm{fs}]$ & 1.0 \\
Strain rate $[1 / \mathrm{ns}]$ & 50.0 \\
\hline
\end{tabular}

the situation obtained for the selected atom with that of atom in reference crystal structure, that atom is identified with some crystal structure. By using conventional version of CNA, an atom is primarily recognized as face-centered cubic (FCC), body-centered cubic (BCC), or HCP structure. If those basic crystals do not match, the atom is categorized as "other structure". In the figures of the present paper, atoms can be visually recognized by color code attributed to each crystal structure identified by CNA as follows: green, blue, pink, or white represents FCC, BCC, HCP, or other structure, respectively. In Figure 1, we show an example of GB model which is already analyzed by CNA. Calculation conditions for MD simulation are shown in Table 2. During relaxation and loading simulations, the system temperature is kept constant at 10 Kelvin.

\subsection{Slip Mechanism of Mg Crystal: Theory, Detection Method.} As mentioned, $\mathrm{Mg}$ crystal preferentially slips on basal plane as schematically shown in Figure 2(a)(A). However, to compensate large strain in plastic regime applied to the system, it may exhibit other shear deformation mechanisms, such as slips activated on other planes (prismatic slip, 1st and 2nd pyramidal slips) or twinning (deformation twinning), as shown in Figures 2(a)(B)-(D) and 2(b). In MD simulations, occurrence of slip or twinning can be detected by change 
TABLE 3: Relationship between GB models and identified deformation mechanisms.

\begin{tabular}{lcc}
\hline Model & Nucleation point of dislocation emission & Identified deformation mechanism \\
\hline A & Edge dislocation & Pyramidal slip \\
B & Grain boundary & Twin \\
C & Grain boundary & Twin \\
D & Grain boundary & Twin \\
E & Grain boundary & Twin \\
F & Grain boundary & Twin \& Basal slip \\
G & Grain boundary & Basal slip \\
H & Grain boundary & Basal slip \\
I & Grain boundary & Basal slip \\
J & In grains & Prismatic slip \\
K & Grain boundary & Prismatic slip \\
L & Grain boundary & Prismatic slip \\
M & Grain boundary & Prismatic slip \\
\hline
\end{tabular}

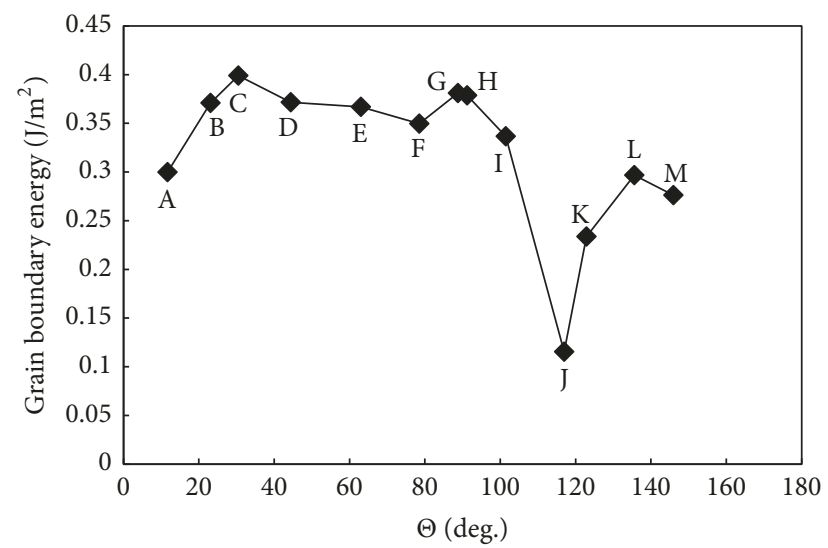

FIGURE 3: Relationship between $\Theta$ and grain boundary energy.

in crystalline identification (HCP, FCC, BCC, or other) which is provided by CNA. Besides, from atomic and local arrangement around such deformation zone, we can visually distinguish those mechanisms presented in Figure 2. Later, in summarizing the present study, we will show correlation between the structural types of GB and those deformation mechanisms. Generally, easiness to occur is recognized as follows: basal slip > twin > prismatic slip > pyramidal slip, though it is likely dependent on temperature condition. We tried to evaluate value and order of stresses or energies required by each mechanism in our GB models. However, clear and unified understanding has not been obtained yet, because of the complicated effect concerning temperature and loading conditions. Therefore, it will need further study and no discussion on it is made in this paper.

\section{Results and Discussion}

3.1. GB Structures and Energies after Structural Relaxation. In Figure 3, the relationship between misorientation angle $\Theta$ and GB energy is shown. The model "J", one of the large angle GBs, has the lowest GB energy of all models. As a result, the energy graph shows one sharp cusp, which is well observed in other pure metals or alloys, via experiment [31] and computation [32]. This indicates that atoms in this GB structure are regularly and compactly arranged on GB plane to exhibit the most stable structure. Metallic GBs often present a special atomic arrangement (called structural unit) around GB plane, usually having a certain periodicity along the plane. The structural units in GB of models " $\mathrm{F}$ " and "J" are shown in Figure 4, for example. It is recognized that the GB structures are formed by periodic structural unit (portion surrounded by solid line in the figure). This result is almost consistent with previous experimental study [7].

In Figure 5, atomic configuration of GB models together with the color corresponding to magnitude of potential energy is shown especially in the vicinity of GB. By comparing GB models shown in Figure 5, it is well recognized that there is a clear difference in the energy distribution, depending on local atomic structure of each GB model. Most GB models include atoms with relatively high energy in the core region. However, the model "J" solely has less energy distribution than others and its GB structure consists only of atoms with relatively low energy. This is a common feature of coherent boundaries in which the GB plane matches low index plane of crystal unit like $\{11 \overline{2} 2\}$ in this case. In such cases, only a limited number of atoms have high energy and are located in the very vicinity of the GB plane, and they enormously dominate the total GB energy.

In contrast, as shown in Figure 6, it is recognized that the GB structure of model " $\mathrm{A}$ " is formed not by periodic GB structure but rather by column of edge dislocations. In general, GBs with less than $\Theta<30$ (deg.) are called lowangle GBs, and those with more than $30^{\circ}$ are high-angle GBs. It is naturally realized that low-angle GBs comprise edge dislocations also in this Mg's case, like other metals.

3.2. Deformation Mechanism (Obtained by Tensile Simulation). In Table 3, relationship between nucleation point of dislocation emission and identified deformation mechanism for each GB models under tensile loading is summarized. For model " $A$ ", in earlier stage, edge dislocations have already 


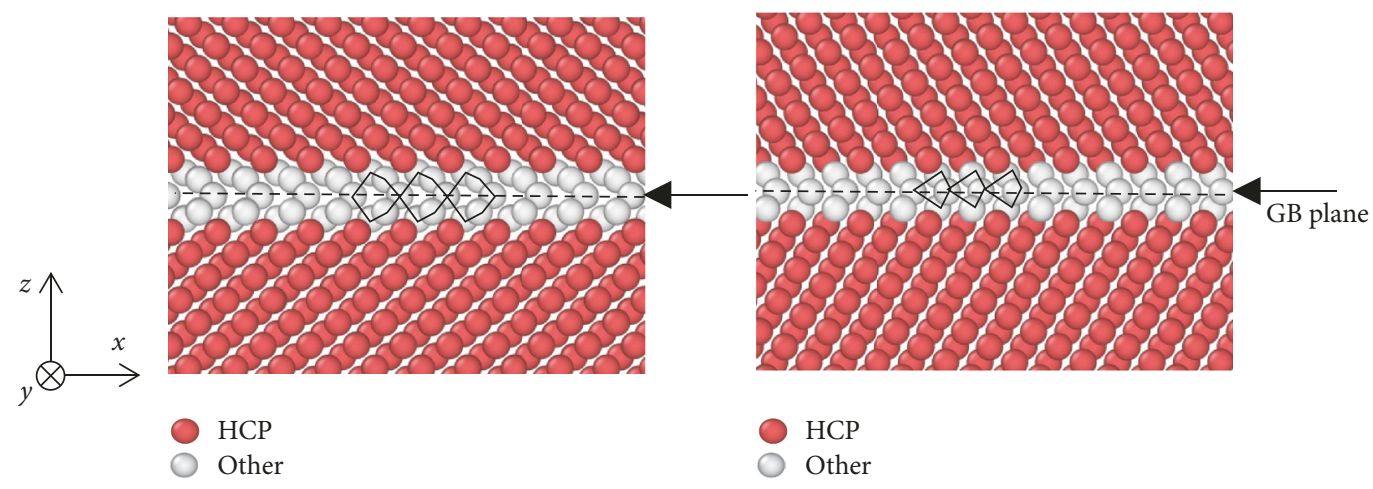

(a)

(b)

FIGURE 4: Example of the structural unit of GB for (a) model F and (b) model J.
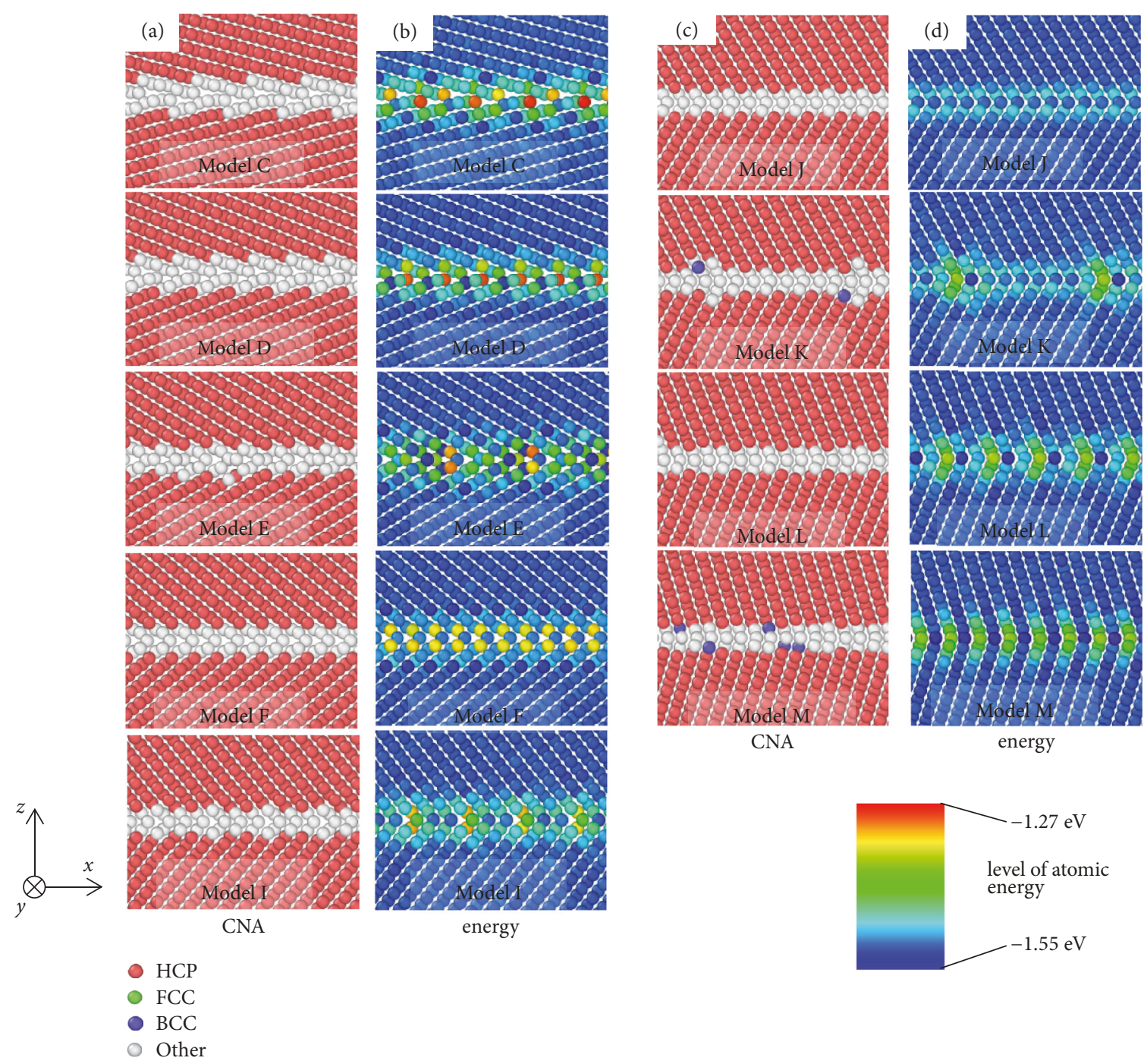

CNA

energy

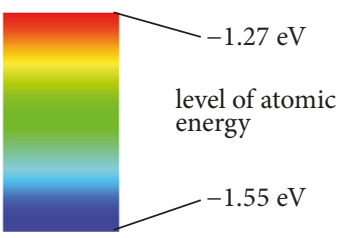

FIGURE 5: GB structures analyzed by CNA and potential energy attributed to each atom for models C, D, E, F, I ((a) and (b)) and for models J, K, L, M ((c) and (d)), respectively. 


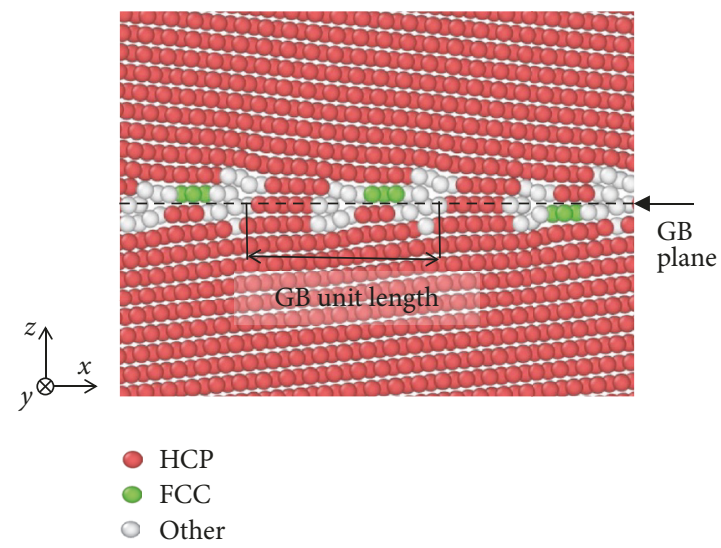

FIgURE 6: GB structure consisting of edge dislocations (analyzed by CNA, for model A).

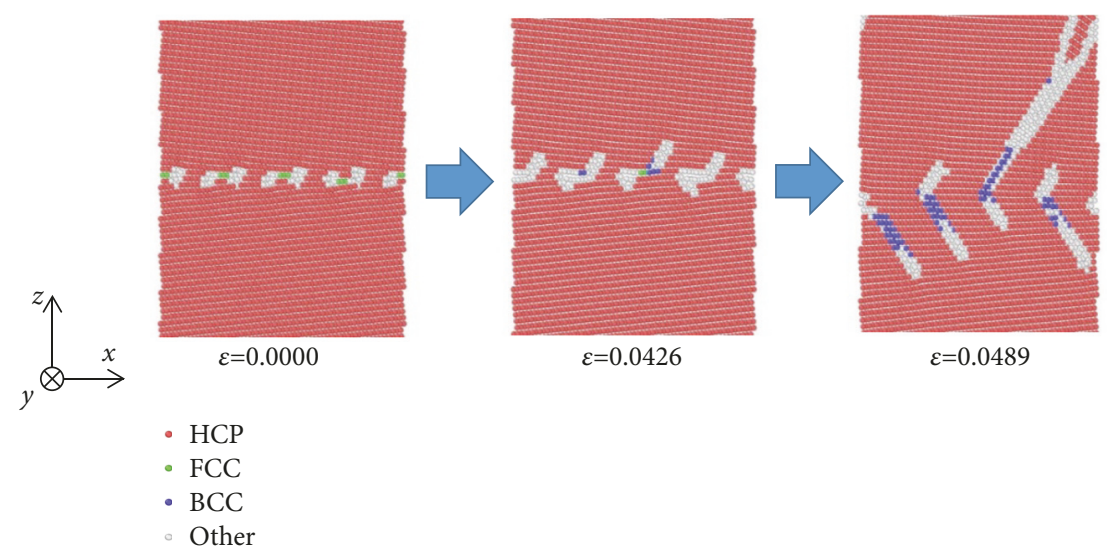

FIGURE 7: Deformation process during tensile test analyzed by CNA (model A; $\varepsilon$ is tensile strain in $z$ direction).

been aligned in parallel fashion when the structural relaxation process is completed. However, after these linear defects enter in the direction of secondary pyramidal slip, their propagation route slightly deviates from it. Then, mismatched displacement of atoms around slip tends to be accumulated along the pyramidal slip plane. Consequently, this situation causes the Mg crystal to generate deformation twinning as shown in Figure 7. From this result, it is understood that twinning by shear is easier to occur than pyramidal slip.

In models "D", "F", and "I", as category of high-angle $\mathrm{GB}$, atoms inside $\mathrm{GB}$ region are rearranged and result in new dislocation emission. An example of this mechanism can be seen in Figure 8. During loading, atoms with relatively high potential energy slightly alter their positions to reduce their energy via a kind of collaborative move with other neighboring atoms. However, due to these rearranging moves, the GB region itself is given more crystalline mismatch and strain energy eventually and should deform in plastic regime, releasing dislocations, generating slip, and making deformation twin in its attached grains.

In other models ("B”, “C”, “E”, “G”, “ $H$ ”, “K”, “L”, and " $\mathrm{M}$ "), dislocations are also released from the GB region. However, in these models, it is likely that any process of dislocation emission does not affect the equilibrium structure of the GB in itself. A visible example of this process is shown in Figure 9. Unfortunately, dislocation releasing mechanism of these models is still unclear and needs more scrutiny.

Only model "J", as shown in Figure 10, shows that any dislocation is not released from GB region, but from inside of the grain. As we mentioned in previous section, the GB energy of the model "J" is particularly low (i.e., the GB structure of the model " $\mathrm{J}$ " is the most stable of all models). Therefore, the GB region has no energetic profit for creating a nucleation point for defect, that is, origin for dislocation emission or twinning.

\section{Conclusion}

In the present work, we created grain boundary (GB) models of hexagonal $\mathrm{Mg}$ crystal for molecular dynamics (MD) simulation, in which the coincidence site lattice (CSL) theory with axis of rotation [1 $1 \overline{100}$ ] was developed and their GB energies were evaluated. We also conducted observation on deformation mechanism of GBs under uniaxial tensile loading by using MD method.

From GB structures after relaxation calculation, we obtained correlation between the energy and the structure of each GB. Typical difference in local arrangement of atoms 


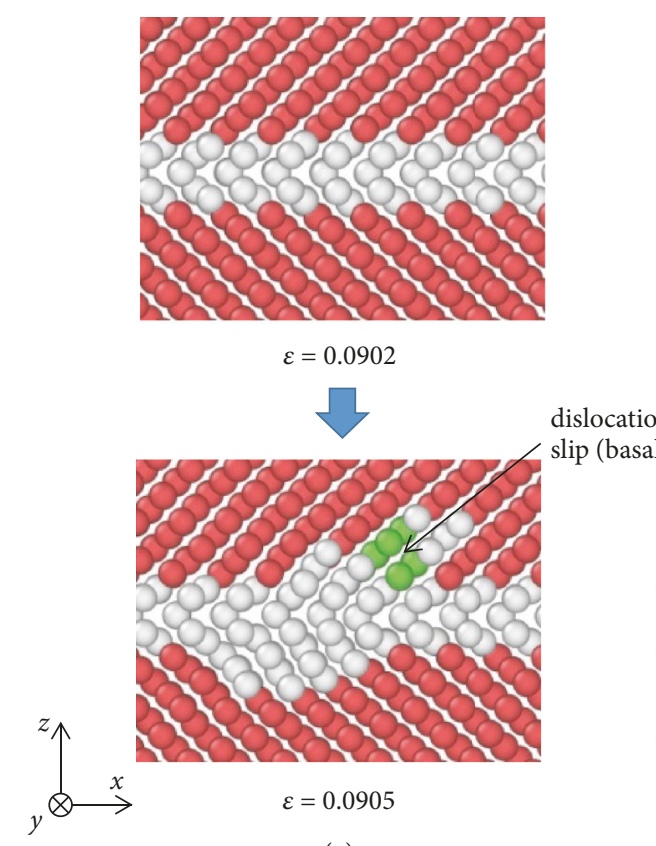

(a)

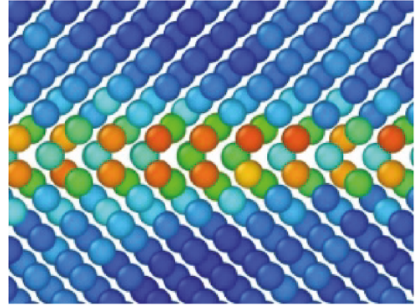

$\varepsilon=0.0902$

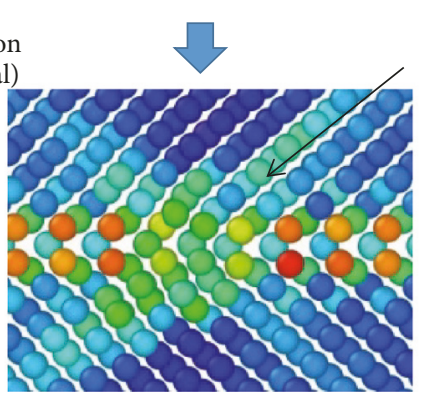

$\varepsilon=0.0905$

(b)

FIGURE 8: Deformation process during tensile test for (a) analyzed by CNA. (b) Potential energy distribution (model F; $\varepsilon$ is tensile strain in $z$ direction).

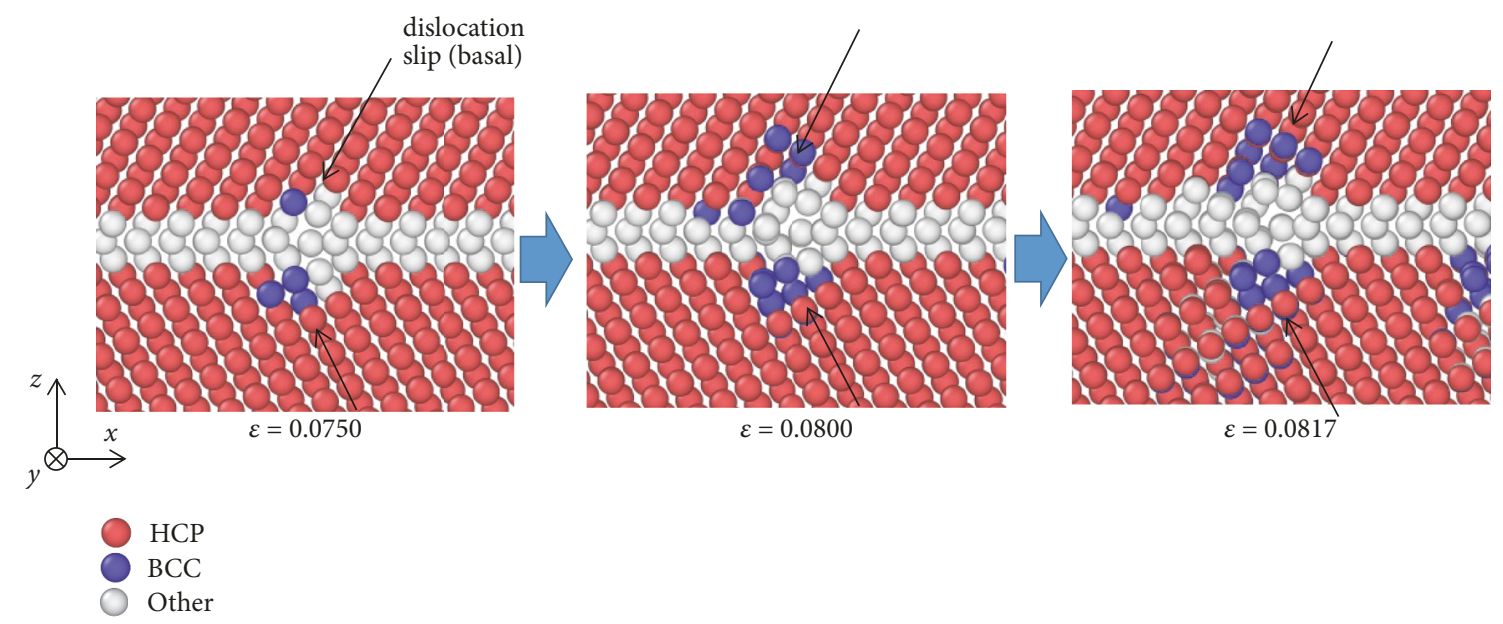

Figure 9: Deformation process during tensile test (model $\mathrm{K} ; \varepsilon$ is tensile strain in $z$ direction).

composing GB structures is found between low- and highangle GBs, like other metals. In particular, some GBs possess a special atomic arrangement (structural unit) around the GB plane with some periodicity.

For tensile simulations, we also found a difference in dislocation emission mechanisms between low- and highangle GBs. High-angle GBs work as a nucleation point of dislocation emission, but there is a special GB in which slip occurs rather inside grains, not in $\mathrm{GB}$ region.

Those results are for relatively simple GB structures, so there should be many other asymmetrical or threedimensionally connected curved GBs in real system. Polycrystalline GB models will serve somewhat other insights [23], but it is left to further studies. Besides, the authors think that shearing deformation with GB structures should be focused on in their future work. This work, we believe, is meaningful in order to step further the research toward complicated GB structures of Mg crystal and their mechanical properties.

\section{Data Availability}

The data used to support the findings of this study are available from the corresponding author upon request.

\section{Conflicts of Interest}

The authors declare that they have no conflicts of interest. 


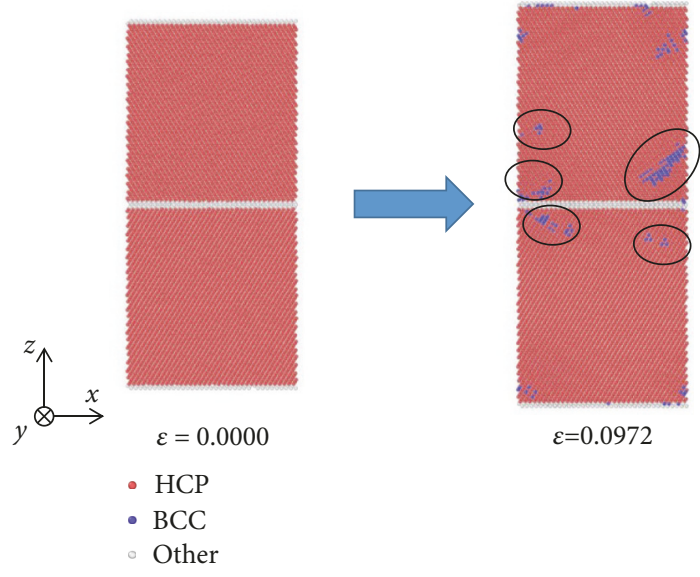

FIGURE 10: Deformation process during tensile test (model J; $\varepsilon$ is tensile strain in $z$ direction. Marked regions are twins found inside grains.)

\section{Acknowledgments}

This work was supported by JSPS KAKENHI Grant no. 16K05994 (2016-2018, Grant-in-Aid for Scientific Research (C)) and by the Kansai University Grant-in-Aid for Progress of Research in graduate course, 2017 (Apr)-2018 (Mar). The authors also acknowledge the financial support from Nippon Steel \& Sumitomo Metal Corporation.

\section{References}

[1] B. L. Mordike and T. Ebert, "Magnesium Properties-applications-potential," Materials Science and Engineering: A Structural Materials: Properties, Microstructure and Processing, vol. 302, no. 1, pp. 37-45, 2001.

[2] H. Yoshinaga and R. Horiuchi, "On the nonbasal slip in magnesium crystals," Transactions of the Japan Institute of Metals, vol. 5, no. 1, pp. 14-21, 1964.

[3] G. Nussbaum, P. Sainfort, G. Regazzoni, and H. Gjestland, "Strengthening mechanisms in the rapidly solidified AZ 91 magnesium alloy," Scripta Materialia, vol. 23, no. 7, pp. 10791084, 1989.

[4] N. Tsuji, Y. Ito, Y. Saito, and Y. Minamino, "Strength and ductility of ultrafine grained aluminum and iron produced by ARB and annealing," Scripta Materialia, vol. 47, no. 12, pp. 893899, 2002.

[5] M. Kai, Z. Horita, and T. G. Langdon, "Developing grain refinement and superplasticity in a magnesium alloy processed by high-pressure torsion," Materials Science and Engineering: A Structural Materials: Properties, Microstructure and Processing, vol. 488, no. 1-2, pp. 117-124, 2008.

[6] H. Miura, G. Yu, and X. Yang, "Multi-directional forging of AZ61Mg alloy under decreasing temperature conditions and improvement of its mechanical properties," Materials Science and Engineering: A Structural Materials: Properties, Microstructure and Processing, vol. 528, no. 22-23, pp. 6981-6992, 2011.

[7] J. T. Chou, K. Ikeda, and H. Nakashima, "Energy and structure of [1100] symmetric tilt grain boundaries in magnesium," Journal of the Japan Institute of Metals and Materials, vol. 69, no. 3, pp. 303-307, 2005 (Japanese).
[8] M. Uranagase and R. Matsumoto, “Tension-compression asymmetry in uniaxial deformation of a magnesium bicrystal with symmetric tilt grain boundary," Computational Materials Science, vol. 118, pp. 124-132, 2016.

[9] I. J. Beyerlein, L. Capolungo, P. E. Marshall, R. J. McCabe, and C. N. Tomé, "Statistical analyses of deformation twinning in magnesium," Philosophical Magazine, vol. 90, no. 16, pp. 21612190,2010

[10] Q. Yu, L. Qi, K. Chen, R. K. Mishra, J. Li, and A. M. Minor, “The nanostructured origin of deformation twinning," Nano Letters, vol. 12, no. 2, pp. 887-892, 2012.

[11] R. Sánchez-Martín, M. T. Pérez-Prado, J. Segurado et al., "Measuring the critical resolved shear stresses in $\mathrm{Mg}$ alloys by instrumented nanoindentation," Acta Materialia, vol. 71, pp. 283-292, 2014.

[12] R. Sánchez-Martín, M. Pérez-Prado, J. Segurado, and J. MolinaAldareguia, "Effect of indentation size on the nucleation and propagation of tensile twinning in pure magnesium," Acta Materialia, vol. 93, pp. 114-128, 2015.

[13] J. Wang, I. J. Beyerlein, and C. N. Tomé, "An atomic and probabilistic perspective on twin nucleation in Mg," Scripta Materialia, vol. 63, no. 7, pp. 741-746, 2010.

[14] M. Pozuelo, S. N. Mathaudhu, S. Kim, B. Li, W. H. Kao, and J.-M. Yang, "Nanotwins in nanocrystalline Mg-Al alloys: An insight from high-resolution TEM and molecular dynamics simulation," Philosophical Magazine Letters, vol. 93, no. 11, pp. 640-647, 2013.

[15] B. Li and E. Ma, "Atomic shuffling dominated mechanism for deformation twinning in magnesium," Physical Review Letters, vol. 103, article 035503, 2009.

[16] B. Li and E. Ma, "Pyramidal slip in magnesium: Dislocations and stacking fault on the 1011 plane," Philosophical Magazine, vol. 89, no. 14, pp. 1223-1235, 2009.

[17] R. Aghababaei and S. P. Joshi, "Micromechanics of tensile twinning in magnesium gleaned from molecular dynamics simulations," Acta Materialia, vol. 69, pp. 326-342, 2014.

[18] J. Wang, Q. Yu, Y. Jiang, and I. J. Beyerlein, "Twinningassociated boundaries in hexagonal close-packed metals," JOM: The Journal of The Minerals, Metals \& Materials Society (TMS), vol. 66, no. 1, pp. 95-101, 2014.

[19] H. Zhang, "Atomistic simulation of sliding of [1010] tilt grain boundaries in Mg," Journal of Materials Research, vol. 24, no. 11, pp. 3446-3453, 2009.

[20] D.-H. Kim, M. V. Manuel, F. Ebrahimi, J. S. Tulenko, and S. R. Phillpot, "Deformation processes in [1120̄]-textured nanocrystalline $\mathrm{Mg}$ by molecular dynamics simulation," Acta Materialia, vol. 58, no. 19, pp. 6217-6229, 2010.

[21] D. H. Kim, F. Ebrahimi, M. V. Manuel, J. S. Tulenko, and S. R. Phillpot, "Grain-boundary activated pyramidal dislocations in nano-textured $\mathrm{Mg}$ by molecular dynamics simulation," Materials Science and Engineering: A Structural Materials: Properties, Microstructure and Processing, vol. 528, no. 16-17, pp. 5411-5420, 2011.

[22] C. D. Barrett, H. El Kadiri, and M. A. Tschopp, "Breakdown of the Schmid law in homogeneous and heterogeneous nucleation events of slip and twinning in magnesium," Journal of the Mechanics and Physics of Solids, vol. 60, no. 12, pp. 2084-2099, 2012.

[23] H. Y. Song and Y. L. Li, "Atomic simulations of effect of grain size on deformation behavior of nano-polycrystal magnesium," Journal of Applied Physics, vol. 111, article 044322, 2012. 
[24] M. S. Daw and M. I. Baskes, "Embedded-atom method: derivation and application to impurities, surfaces, and other defects in metals," Physical Review B: Condensed Matter and Materials Physics, vol. 29, no. 12, pp. 6443-6453, 1984.

[25] X.-Y. Liu, J. B. Adams, F. Ercolessi, and J. A. Moriarty, "EAM potential for magnesium from quantum mechanical forces," Modelling and Simulation in Materials Science and Engineering, vol. 4, no. 3, pp. 293-303, 1996.

[26] W. Bollmann, "On the geometry of grain and phase boundaries I. General Theory," Philosophical Magazine, vol. 16, no. 140, pp. 363-381, 1967.

[27] K. Saitoh, Doctoral thesis, Osaka University Knowledge Archive, 1996 (Japanese), in Japanese.

[28] T. Hondoh and A. Higashi, "A grain boundary model of hexagonal crystals/with special reference to the G. B. properties in ice," Journal of the Crystallographic Society of Japan, vol. 22, pp. 270-281, 1980 (Japanese).

[29] K. Saitoh and W. K. Liu, "Molecular dynamics study of surface effect on martensitic cubic-to-tetragonal transformation in $\mathrm{Ni}$ Al alloy," Computational Materials Science, vol. 46, no. 2, pp. 531544, 2009.

[30] J. D. Honeycutt and H. C. Andersen, "Molecular dynamics study of melting and freezing of small Lennard-Jones clusters," The Journal of Physical Chemistry C, vol. 91, no. 19, pp. 4950-4963, 1987.

[31] T. Watanabe, "The impact of grain boundary character distribution on fracture in polycrystals," Materials Science and Engineering: A Structural Materials: Properties, Microstructure and Processing, vol. 176, no. 1-2, pp. 39-49, 1994.

[32] F. Yazdandoost and R. Mirzaeifar, "Tilt grain boundaries energy and structure in NiTi alloys," Computational Materials Science, vol. 131, pp. 108-119, 2017. 


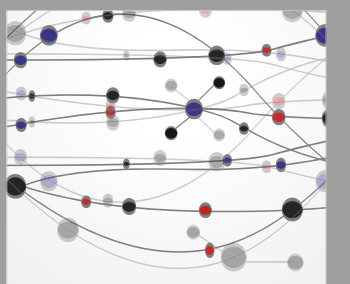

The Scientific World Journal
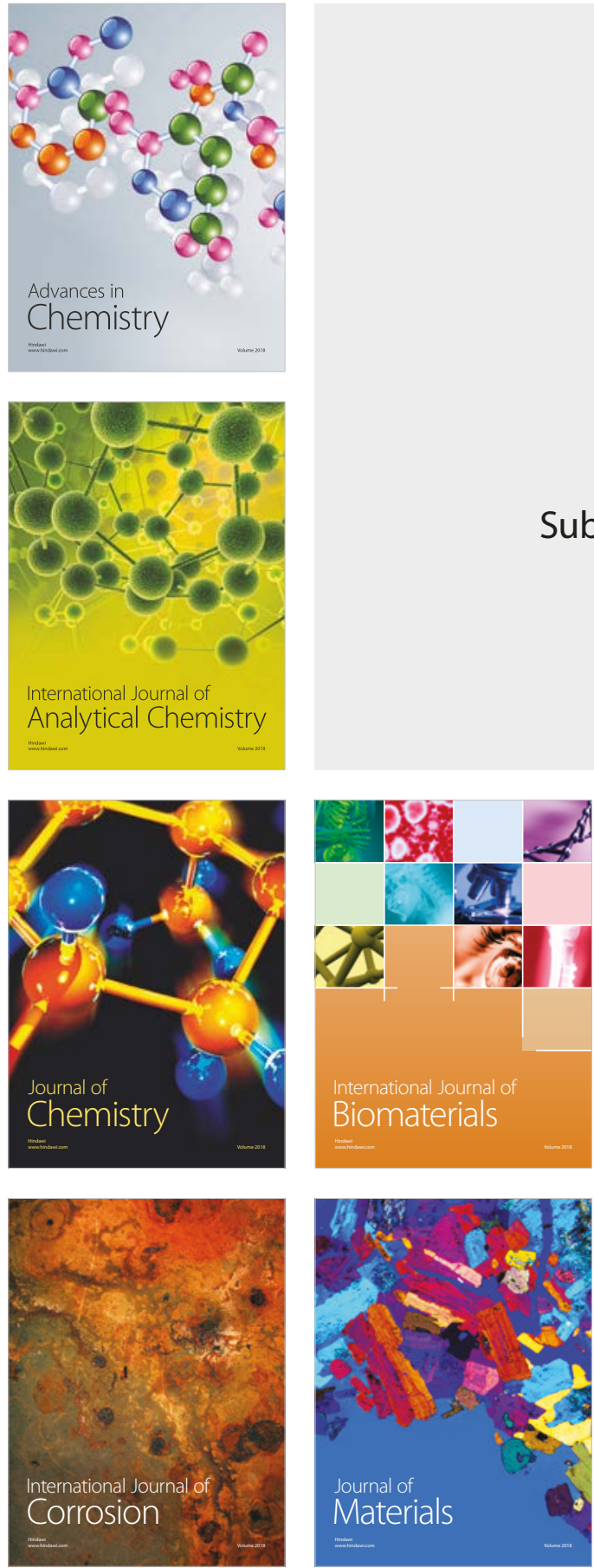

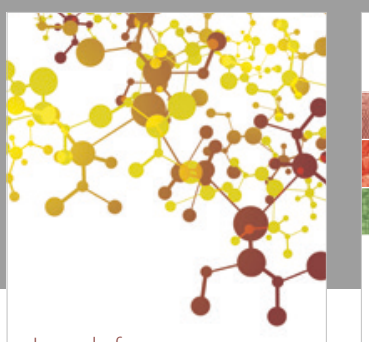

Journal of

Applied Chemistry
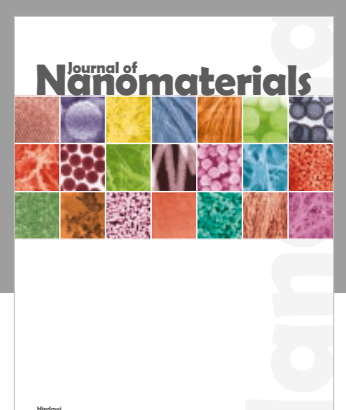

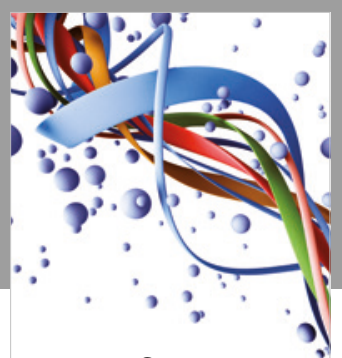

Scientifica

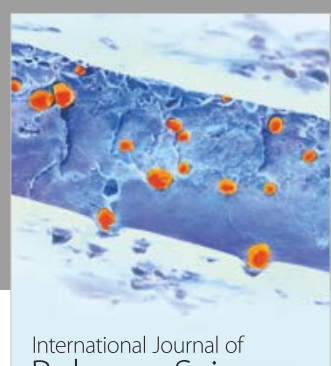

Polymer Science

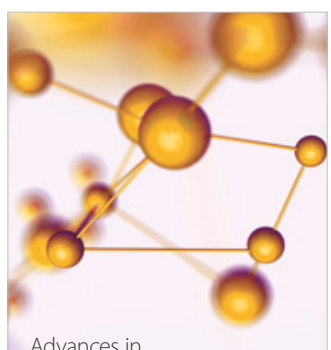

Physical Chemistry
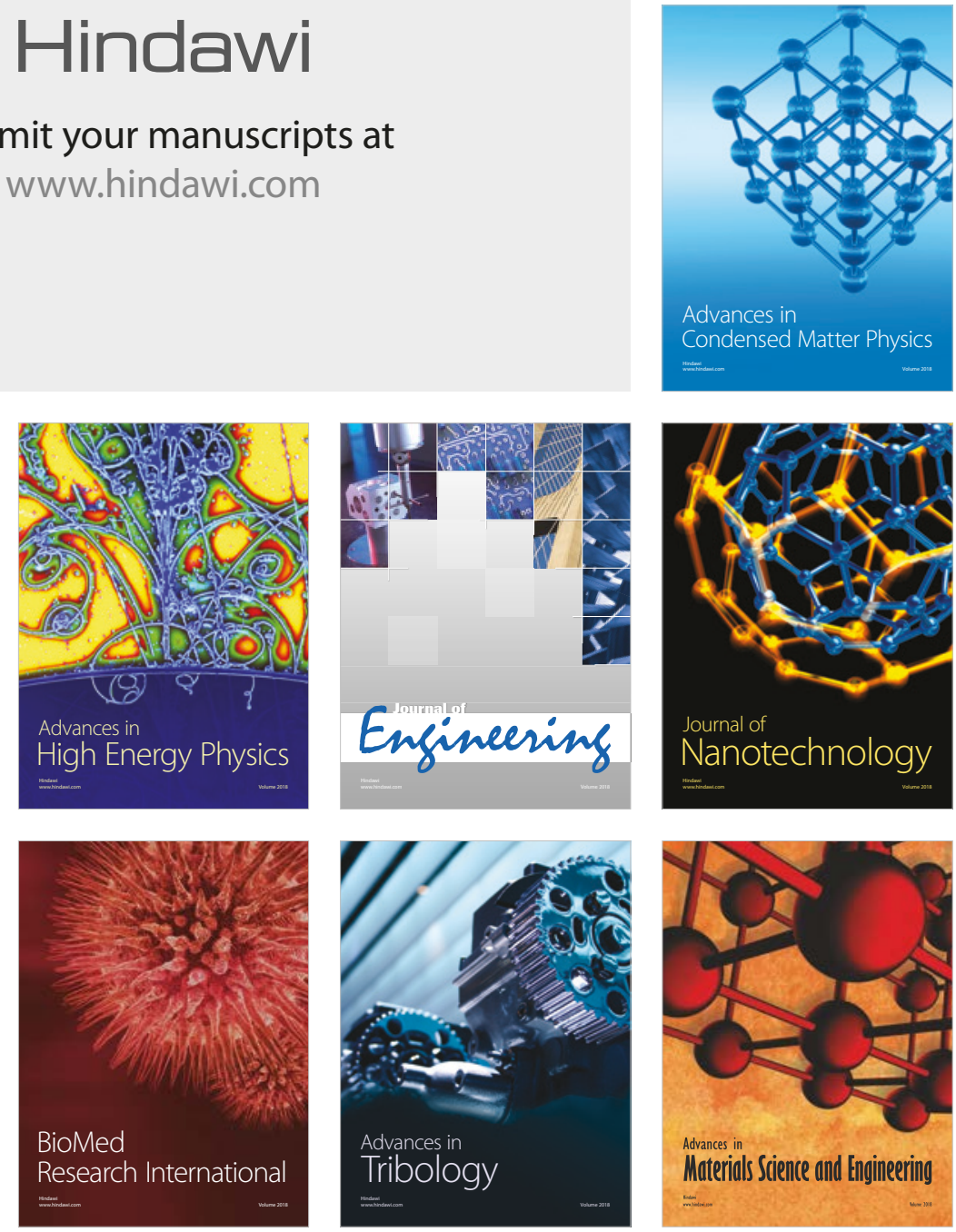